\title{
BMJ Open Protocol, rationale and design of DAbigatran for Stroke PreVention In Atrial Fibrillation in MoDerate or Severe Mitral Stenosis (DAVID-MS): a randomised, open-label study
}

Mi Zhou (D) , ${ }^{1}$ Esther W Chan, ${ }^{2}$ Jo Jo Hai, ${ }^{1}$ Chun Ka Wong (D) , ${ }^{1}$ Yuk Ming Lau, ${ }^{1}$ Duo Huang, ${ }^{1}$ Cheung Chi Lam, ${ }^{1}$ Chor Cheung Frankie Tam, ${ }^{1}$ Yiu Tung Anthony Wong, ${ }^{1}$ See Yue Arthur Yung, ${ }^{1}$ Ki Wan Kelvin Chan, ${ }^{1}$ Yingqing Feng, ${ }^{3}$ Ning Tan, ${ }^{3}$ Ji-yan Chen, ${ }^{3,4}$ Chi Yui Yung, ${ }^{5}$ Kwok Lun Lee, ${ }^{5}$ Chun Wai Choi, ${ }^{6}$ Ho Lam, ${ }^{6}$ Andrew Ng, ${ }^{7}$ Katherine Fan, ${ }^{7}$ Man Hong Jim, ${ }^{7}$ Kai Hang Yiu, ${ }^{1}$ Bryan P. Yan, ${ }^{8}$ Chung Wah Siu ${ }^{1}$

To cite: Zhou M, Chan EW, Hai JJ, et al. Protocol, rationale and design of DAbigatran for Stroke PreVention In Atrial Fibrillation in MoDerate or Severe Mitral Stenosis (DAVID-MS): a randomised, open-label study. BMJ Open 2020;10:e038194. doi:10.1136/ bmjopen-2020-038194

- Prepublication history for this paper is available online. To view these files, please visit the journal online (http://dx.doi org/10.1136/bmjopen-2020038194).

Received 04 March 2020 Revised 21 June 2020 Accepted 29 July 2020

Check for updates

(c) Author(s) (or their employer(s)) 2020. Re-use permitted under CC BY-NC. No commercial re-use. See rights and permissions. Published by BMJ.

For numbered affiliations see end of article.

Correspondence to Dr Chung Wah Siu; cwdsiu@hku.hk

\section{ABSTRACT}

Introduction Current international guidelines recommend non-vitamin K oral anticoagulants (NOACs) for stroke prevention among patients with non-valvular atrial fibrillation (AF) at significant ischaemic stroke risk given the superior safety and comparable efficacy of NOACs over warfarin. Nonetheless, the safety and effectiveness of NOACs have not been evaluated in patients with AF with underlying moderate or severe mitral stenosis (MS), hence the recommended stroke prevention strategy remains warfarin therapy.

Method and analysis MS remains disproportionately prevalent in Asian countries compared with the developed countries. This prospective, randomised, open-label trial with blinded endpoint adjudication aims to evaluate the safety and efficacy of dabigatran for stroke prevention in AF patients with moderate or severe MS. Patients with AF aged $\geq 18$ years with moderate or severe MS not planned for valvular intervention in the coming 12 months will be randomised in a 1:1 ratio to receive dabigatran $110 \mathrm{mg}$ or $150 \mathrm{mg}$ two times per day or warfarin with international normalised ratio $2-3$ in an open-label design. Patients with estimated creatinine clearance $<30 \mathrm{~mL} /$ min, or with a concomitant indication for antiplatelet therapy will be excluded. The primary outcome is a composite of stroke and systemic embolism. Secondary outcomes are ischaemic stroke, systemic embolism, haemorrhagic stroke, intracranial haemorrhage, major bleeding and death. The estimated required sample size is approximately 686 participants.

Ethics and dissemination The study protocol has been approved by the Institutional Review Board of the University of Hong Kong and Hong Kong West Cluster, Hospital Authority, Hong Kong for Fung Yiu King Hospital, Grantham Hospital, Queen Mary Hospital and Tung Wah Hospital in Hong Kong. Results will be published in peerreviewed journals.

Trial registration number ClinicalTrials.gov Registry (NCT04045093); pre-results.
Strengths and limitations of this study

- This is the first investigator-initiated, open-label, randomised clinical trial to compare the effectiveness and safety of dabigatran and warfarin therapy for stroke prevention in patients with atrial fibrillation (AF) and moderate or severe mitral stenosis (MS).

- The study is designed to provide clinicians with robust, much-needed information regarding stroke prevention strategy for patients with AF and moderate or severe MS.

- The results of study will have immediate and longterm impacts on the management of these very high-risk patients with AF.

- This study will provide the necessary evidence for establishing international clinical practice guidelines for stroke prevention in patients with AF and MS.

- Since the clinical trial will be conducted mainly in Hong Kong and Mainland China, it is expected that most recruited subjects will be of Chinese ethnicity, which may limit generalisability of the trial results.

\section{INTRODUCTION}

Atrial fibrillation (AF) is the most common sustained cardiac arrhythmia encountered in clinical practice. ${ }^{1}$ Patients with $\mathrm{AF}$ are at increased risk of ischaemic stroke and systemic thromboembolism due to the formation and embolism of left atrial thrombi, ${ }^{1-3}$ hence long-term oral anticoagulant for thromboprophylaxis is the cornerstone in AF management. In previous randomised clinical trials in the last century, warfarin has been shown to be highly effective in reducing stroke risk compared with placebo by as much as $64 \%$ in patients with $\mathrm{AF}^{4}$ More recently, non-vitamin 
K oral anticoagulants (NOACs) have consistently demonstrated to be safer and more effective for stroke prevention in patients with non-valvular AF compared with warfarin and have become the recommended standard of care for the management of stroke prevention in nonvalvular AF.

While the stroke risk among patients with AF appears heterogeneous, ${ }^{56}$ patients with underlying valvular heart disease, particularly mitral stenosis (MS) are at very high risk for stroke with an annual stroke risk ranging from $4 \%$ to $17 \%$ if left un-anticoagulated ${ }^{7}$ and the highest recurrences. ${ }^{8}$ However, patients with $\mathrm{AF}$ and underlying MS are typically excluded in randomised control trials. ${ }^{9}$ As a result, current international guidelines for management of AF do not recommend NOACs for stroke prevention in patients with $\mathrm{AF}$ and underlying moderate or severe MS. ${ }^{3}$ Nonetheless, off-label use of NOAC in patients with $\mathrm{AF}$ and MS is not uncommon in the real-world practice. In a recently published retrospective, observational analysis from the Republic of Korea ${ }^{10}$ in a cohort of 7357 patients with MS receiving anticoagulation therapy, 35\% of these patients were in fact treated with NOAC with the remaining $65 \%$ with warfarin. More importantly, after propensity matching, it was shown that patients treated with NOAC had a substantially lower risk of ischaemic stroke/systemic embolism with an annualised risk of $2.22 \%$ /year, compared with that of $4.19 \% /$ year for patients treated with warfarin, (adjusted HR: 0.28 ; $95 \%$ CI: 0.18-0.45), suggesting a potential role of NOAC among patients with AF and underlying MS. ${ }^{8} 1011$

This is of particular importance for Asian patients with $\mathrm{AF}$, in whom MS remains relatively prevalent despite a declining trend. ${ }^{7}$ More importantly, the much higher baseline risk of intracranial haemorrhage and apparently higher ischaemic stroke risk in Asian populations potentially undermine the benefits of warfarin therapy. ${ }^{12-14}$ Notably, compared with warfarin, the effectiveness and safety of NOACs appear to be even more superior in Asian populations than Caucasian populations as shown in subanalyses of pivotal randomised control trials ${ }^{15-17}$ as well as in studies using real-world data. ${ }^{18-23}$ To our knowledge, this is the first multicentre randomised control trial aims to comparing NOAC with warfarin to address the knowledge in stroke prevention strategy in patients with $\mathrm{AF}$ and moderate or severe MS. This will have immediate and long-term impacts on the management of these very high-risk patients with AF.

\section{METHODS AND ANALYSIS}

This clinical trial protocol follows the Standard Protocol Items: Recommendations for Interventional Trials. ${ }^{24}{ }^{25}$ The underlying protocol follows the Consolidated Standards of Reporting Trials. ${ }^{26} 27$

\section{Study design}

This is an investigator-initiated, open-label randomised clinical trial to compare the effectiveness and safety of

\section{Box 1 Inclusion and exclusion criteria}

\section{Inclusion criteria}

- Patients with AF documented with standard 12-lead ECG documented AF on the day of screening or randomisation.

- Patients with age $>18$ years.

- Patients with moderate or severe MS that is, MVA $<1.5 \mathrm{~cm}^{2}$.

- Patients should be able to provide a written, informed consent.

- Patients should have all four inclusion criteria fulfilled to be qualified for the study.

Exclusion criteria

- Patients with prosthetic valve or with active endocarditis.

- Patients with heart failure symptom.

- Patients with planned valvular intervention within 1 year.

- Patients with left atrial appendage occlusive device.

- Patients with planned AF ablation.

- Patients with a history of intracranial, intraocular, spinal or retroperitoneal bleeding.

- Unexplained anaemia (haemoglobin level $<100 \mathrm{~g} / \mathrm{L}$ ) or thrombocytopenia (platelet count $<100 \times 10^{9} / \mathrm{L}$ ).

- Need for anticoagulant therapy of disorders other than AF.

- Patients receiving antiplatelet therapy for disorders other than AF.

- Patients receiving concomitant P-gp inhibitors and/or medications known to interact with dabigatran.

- Uncontrolled hypertension (systolic blood pressure $>180 \mathrm{~mm} \mathrm{Hg}$ and/or diastolic blood pressure $>100 \mathrm{~mm} \mathrm{Hg}$ ).

- Estimated creatinine clearance $\leq 30 \mathrm{~mL} / \mathrm{min}$.

- Liver dysfunction of Child-Pugh stage B or C.

- Women who are pregnant or of childbearing potential who refuse to use a medically acceptable form of contraception throughout the study.

- Patients considered unreliable by the investigator or have a life expectancy less than 1 yearbecause of concomitant disease, or has any condition, which in the opinion of the investigator, would not allow safe participation in the study (eg, drug addiction, alcohol abuse).

AF, atrial fibrillation; MS, mitral stenosis; MVA, mitral valvular area; P-gp, Pglycoprotein.

dabigatran $150 \mathrm{mg}$ or $110 \mathrm{mg}$ two times per day according to kidney function with warfarin therapy with target international normalised ratio (INR) 2-3 for stroke prevention in patients with AF and moderate or severe MS.

\section{Study participants}

Patients will be recruited from participating specialist cardiology centres in Hong Kong Special Administrative Region (SAR) of China and Mainland China. Written informed consent will be obtained from all study participants. Box 1 summarises the inclusion and exclusion criteria for the study. In brief, patients with no symptoms, aged 18 years or above will be eligible if they have AF documented on standard 12-lead ECG performed at screening or randomisation and moderate or severe MS as defined as the mitral valvular area of $1.0-1.5 \mathrm{~cm}^{2}$ and $<1.0 \mathrm{~cm}^{2}$, respectively. Reasons for exclusion include the presence of symptoms, prosthetic valve, left atrial appendage occlusive device and/or active endocarditis; planned valvular intervention and/or planned AF ablation; history of major bleeding including intracranial, intraocular, spinal 


\section{Dabigatran 110/150mg BD}

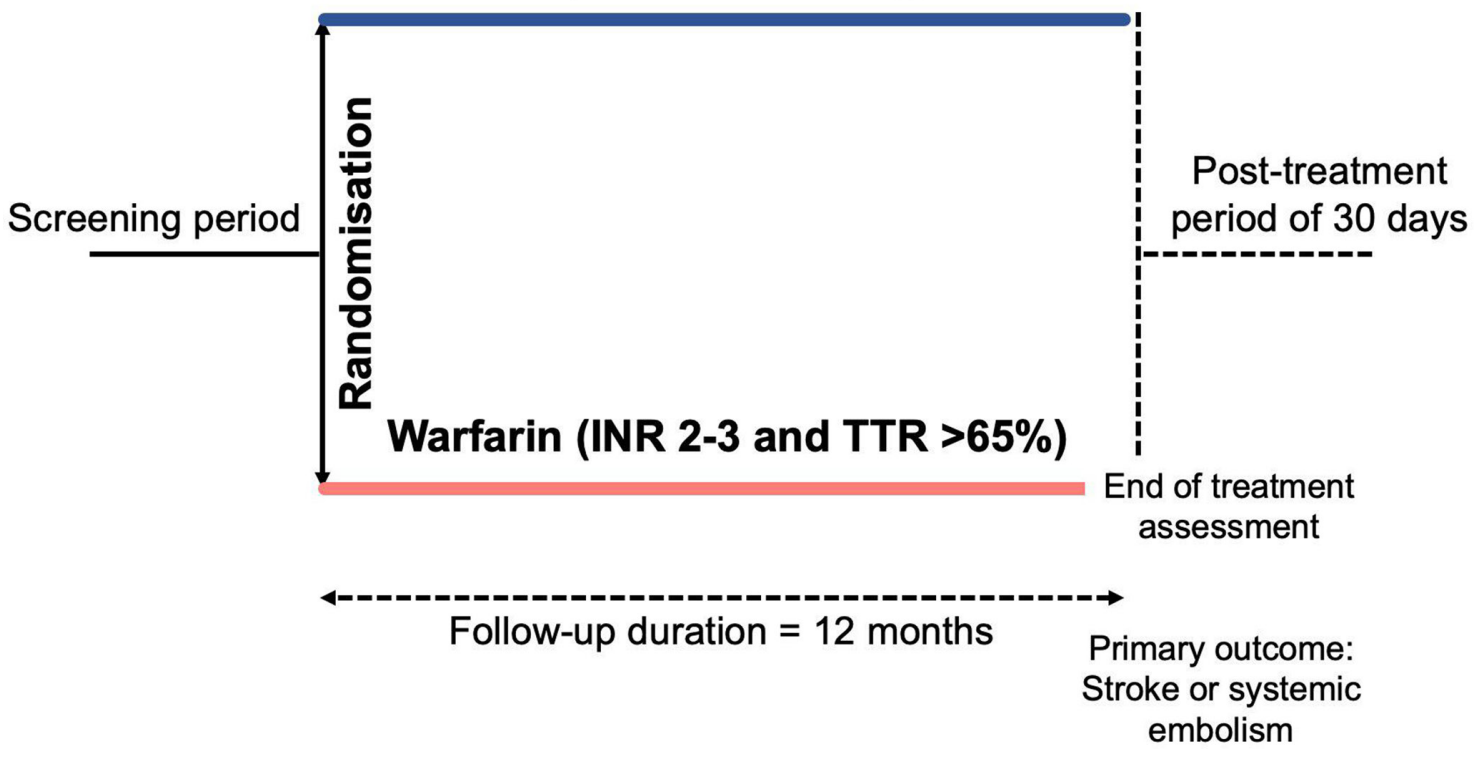

Figure 1 Design of the DAVID-MS Study. BD, two times per day; DAVID-MS, DAbigatran for Stroke PreVention In Atrial Fibrillation in MoDerate or Severe Mitral Stenosis; INR, international normalised ratio; TTR, time in therapeutic range.

or retroperitoneal haemorrhage; unexplained anaemia with haemoglobin level $<100 \mathrm{~g} / \mathrm{L}$ or thrombocytopenia with platelet count $<100 \times 10^{9} / \mathrm{L}$; need for anticoagulant or antiplatelet therapy of conditions other than AF; concomitant use of potent P-glycoprotein inhibitor(s) or drugs with a known interaction with dabigatran; uncontrolled hypertension; significant kidney impairment with estimated creatinine clearance $(\mathrm{CrCl}) \leq 30 \mathrm{~mL} / \mathrm{min}$ by the Cockcroft-Gault Formula ${ }^{28}$; liver dysfunction of ChildPugh stage $\mathrm{B}$ or $\mathrm{C}^{29}$; pregnancy or if there is childbearing potential during the full duration of the study. In addition, patients considered unsuitable by the investigator including short life expectancy $<1$ year due to concomitant disease, substance and/or alcohol abuse or other medical conditions.

\section{Study procedures}

After providing written informed consent, all study participants will be randomly assigned to receive dabigatran or to receive warfarin. The procedure of the trial is summarised in figure 1, the trial will primarily be conducted in Hong Kong and Mainland China. In Hong Kong, there is no local guideline on dabigatran dosage in relation to renal function. In Mainland China, dosage reduction to $110 \mathrm{mg}$ two times per day was recommended in patients with $\mathrm{CrCl}$ in the range $30-49 \mathrm{~mL} / \mathrm{min}$. For patients randomised to receive dabigatran, the dosage regimen will be determined according to the respective estimated $\mathrm{CrCl}$ or if concomitantly taking interacting drugs requiring dosage adjustment. Patients with estimated $\mathrm{CrCl}$ above $50 \mathrm{~mL} /$ min will receive dabigatran $150 \mathrm{mg}$ two times per day, whereas those with $\mathrm{CrCl}$ between 30 and $50 \mathrm{~mL} / \mathrm{min}$ will receive dabigatran $110 \mathrm{mg}$ two times per day. For patients previously on warfarin randomised to receive dabigatran, dabigatran will initiate after discontinuation of warfarin with an INR less than or equal to 2. At the end of study, patients randomised to dabigatran will be switched back to warfarin. Warfarin will be initiated 3 days prior to the termination of dabigatran. INR will be checked 5 days after initiation of warfarin, that is, 2 days after termination of dabigatran to minimise the potential impact of remaining dabigatran levels in elevating the INR. On the other hand, for those randomised to receive warfarin, INR will be measured at least every 8 weeks with a target INR of 2.0-3.0. The time in therapeutic range (TTR) will be calculated for each study participant using Rosendaal method, ${ }^{30}$ in which INR will be assumed to change in a linear manner between measurements, and INR values on the days without measurement are interpolated. The percentage of time during which a study participant has an INR within 2.0-3.0 is taken as TTR. The first follow-up visit will be scheduled 14 days after randomisation and then every 4 months during the study period of 1 year (table 1). Criteria for discontinuation or change of allocated treatment include patient request, drug allergy, intolerable adverse drug reaction and development of other contraindication. Patients who are randomised to receive dabigatran would be switched to warfarin if $\mathrm{CrCl}$ is below $30 \mathrm{~mL} / \mathrm{min}$ and/or develop liver dysfunction of Child-Pugh stage B or C.

\section{Outcomes}

The primary outcome is a composite of stroke or systemic embolism at 1 year. Secondary outcomes are ischaemic stroke, systemic embolism, haemorrhagic stroke, intracranial haemorrhage, major bleeding and death at 1 year. Stroke is defined as a neurological deficit of sudden onset that persisted for more than 24 hours and corresponded to a vascular territory that cannot be explained by other causes (such as trauma, infection or vasculitis). Stroke 


\begin{tabular}{|c|c|c|c|c|c|c|c|c|c|c|c|}
\hline Visits & -1 & 0 & 1 & 2 & 3 & 4 & 5 & 6 & 7 & UNS† & EOS $\ddagger$ \\
\hline Weeks & -2 to 0 & 0 & 8 & 16 & 24 & 32 & 40 & 48 & 56 & -- & $56-60$ \\
\hline Inclusion and exclusion criteria & & $x$ & & & & & & & & & \\
\hline Randomisation & & $X$ & & & & & & & & & \\
\hline Physical examination & & $x$ & $x$ & $x$ & $x$ & $x$ & $x$ & $x$ & $X$ & $X$ & $x$ \\
\hline Echocardiography & & $x$ & & & & & & & & & \\
\hline INR & & $x$ & $X^{*}$ & $X^{*}$ & $X^{*}$ & $X^{*}$ & $X^{*}$ & $X^{*}$ & $X^{*}$ & $X^{*}$ & $X$ \\
\hline Renal function & & $x$ & & $x$ & & $x$ & & $x$ & & $x$ & $x$ \\
\hline Drug dispensing & & $x$ & $X$ & $x$ & $x$ & $x$ & $x$ & $x$ & $x$ & & \\
\hline
\end{tabular}

${ }^{*}$ Only for patients randomised to receive warfarin.

†UNS (unplanned visit): $(X)$ the marked item is optimal and performed according to the judgement of researchers.

fEOS (final visit): make arrangement according to the study end time (if there is a visit within 1 month before the end of study, it is regarded as a final visit, but needs to be supplemented with the items required completely).

INR, international normalised ratio.

will be further classified as ischaemic stroke and haemorrhagic stroke according to computerised axial tomography or MRI of the brain. Intracranial haemorrhage (ICH) consists of haemorrhagic stroke (intracerebral haemorrhage and cerebellar haemorrhage), subdural haemorrhage and subarachnoid haemorrhage, and will be confirmed with computerised axial tomography or MRI of the brain. Systemic embolism is defined as an acute vascular occlusion of an extremity or organ other than the brain, documented by imaging, surgery and/or autopsy.

Major bleeding is defined as a drop in the haemoglobin level of at least $20 \mathrm{~g} / \mathrm{L}$, transfusion of at least 2 units of blood or symptomatic bleeding in a critical area or organ. Life-threatening bleeding includes fatal bleeding, symptomatic intracranial bleeding, bleeding with a haemoglobin drop of at least $50 \mathrm{~g} / \mathrm{L}$ or bleeding requiring transfusion of at least 4 units of blood or inotropic agents or requiring surgery. All outcomes will be adjudicated by two independent investigators in a blinded fashion.

\section{Sample size calculation}

The primary analysis is to test whether dabigatran is noninferior to warfarin for ischaemic stroke prevention in patients with AF and moderate or severe MS. The potential for dabigatran to preserve at least $50 \%$ of the effectiveness of warfarin is considered clinically meaningful, as noninferior in patients with $\mathrm{AF}$ and moderate or severe MS. The non-inferiority margin is 1.49 , which is derived from the only observation study comparing vitamin $\mathrm{K}$ antagonist with NOAC in patients with AF and MS. ${ }^{10}$ In the study, the annual ischaemic stroke risk of patients with $\mathrm{AF}$ and MS receiving vitamin K antagonist and NOAC are 4.19\%/ year and 2.22\%/year, respectively. Accordingly, based on the margin of error $(4.66 \%)$ and the current population of Hong Kong (7 500700 ), a sample size of 686 patients (343 patients in the vitamin $\mathrm{K}$ antagonist group and 343 in the dabigatran group) including $10 \%$ attrition would be needed to satisfy the non-inferiority hypothesis with the upper boundary of the one-sided 95\% CI (or equivalent with a $90 \%$ two-sided CI) and the HR of the primary outcome below the non-inferiority margin of 1.49 . Hierarchical analysis for superiority will be performed if noninferiority is established.

\section{Statistical analysis}

Baseline data will be reported as means and SD for continuous data and as numbers and percentages for categorical data. All endpoints will be analysed according to the intention-to-treat principle, with all patients who undergo randomisation included in the analysis. Clinical events that occur after randomisation and until the end of the study (at 1 year or mortality) will be included in the primary analysis of clinical outcomes. A $p$ value $<0.05$ considered as significant. Calculations will be performed using SPSS software (V.12.0).

\section{Randomisation}

Randomisation will be stratified to each study site to account for variations in patient demographics and diagnoses. At each site, patients will be randomised to 'permuted blocks of four' (two of each study arm) to assist in equality of numbers in each arm. An independent research officer who is blinded to this study will generate the random-number table. Study staff responsible for enrolment will be informed of randomisation 
assignment by phone. Subjects and clinicians will not be blinded to the randomisation assignment. Data staff responsible for data entry will be blinded from randomisation assignment.

\section{Data collection and management}

After enrolment, each subject will be assigned a unique identifier to be used in database. Data will be entered by study staff and data accuracy will be verified by study principal investigator. Data quality control measures include queries to identify missing data, outliers and discrepancies. The database will be password protected and encrypted. Only study staff will have access to the database. All paper records will be deidentified and stored securely in a locked cabinet for 5 years. Subjects who withdraw from the study will have continuous monitoring stopped, usual care continued and final outcome collected for analysis.

\section{Data monitoring and safety}

An independent Safety Committee will be established comprising of an emergency clinician, clinical pharmacologist and toxicologist. They will receive regular reports during patient enrolment and be notified of any adverse drug reaction and study protocol violation. The Safety Committee is led by Professor Bernard Cheung from the Clinical Pharmacology, Faculty of Medicine, the University of Hong Kong, Hong Kong SAR, China. For patient safety, discontinuation of the study is at the discretion of the cardiology clinician to enable informed decisions to be made regarding subsequent management and alternative medication use. Any medication or therapy, intervention or procedure thought to be necessary for the safe management of the patient may be administered at the discretion of the managing clinician.

\section{Patient and public involvement}

We received input from clinicians and patients which guided the design of the current study and choice of research questions. No patients were directly involved in the design of the study and choice of outcome measures. No patients will be involved in recruitment or conduct of the study. Results of the study will be disseminated to subjects, the public and the scientific community.

\section{ETHICS AND DISSEMINATION}

This research protocol complies with the Declaration of Helsinki and the International Conference on Harmonisation-Good Clinical Practice. The study protocol has been approved by the Institutional Review Board of the University of Hong Kong and Hong Kong West Cluster, Hospital Authority, Hong Kong for Fung Yiu King Hospital, Grantham Hospital, Queen Mary Hospital and Tung Wah Hospital in Hong Kong. Written informed consents will be obtained from all study participants by study staff responsible for recruitment (online supplemental file 1). Important protocol modifications will be

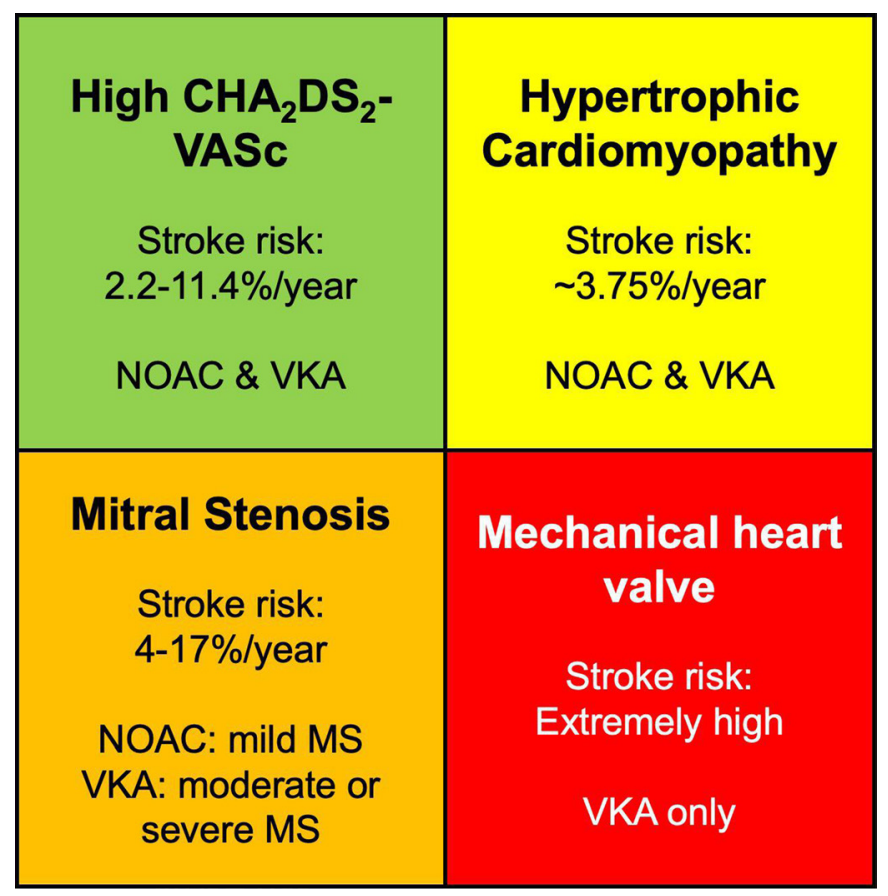

Figure 2 Four main groups of patients with AF requiring long-term anticoagulation therapy. AF, atrial fibrillation; MS, mitral stenosis; NOAC, non-vitamin K oral anticoagulant; VKA, vitamin $\mathrm{K}$ antagonist.

conveyed to investigators, Institutional Review Board, trial registries, regulators, journals and trial participants. After enrolment, each subject will be assigned a unique identifier to be used in database. Personal identity of subjects will not be used for any public purpose, publication or transmitted outside of the study team.

Dataset used during the study will be available from the corresponding author on reasonable request. Collaboration with other investigators will be welcomed. The results of the trial will be published in peer-reviewed journals and presented in conferences.

\section{DISCUSSION}

To our knowledge, this is the first study to evaluate dabigatran as an alternative to warfarin for stroke prevention in patients with AF and moderate or severe MS. The results could fill the gap in stroke prevention strategy for this specific group of patients with $\mathrm{AF}$ with immediate and long-term impacts on clinical practice.

$\mathrm{AF}$ is the most commonly encountered sustained cardiac arrhythmia in clinical practice. ${ }^{131}$ While patients with $\mathrm{AF}$ have in general increased risk of stroke, four subgroups are at particularly high risk necessitating longterm anticoagulation therapy. These include (1) patients with non-valvular $\mathrm{AF}$ and high $\mathrm{CHA}_{2} \mathrm{DS}_{2}$-VASc score, (2) patients with AF and hypertrophic cardiomyopathy, (3) patients with AF and MS, and (4) patients with AF and mechanical heart valves (figure 2). In the past decade, NOAC has emerged as the preferred agent over warfarin for stroke prevention in patients with non-valvular $\mathrm{AF}$ and high $\mathrm{CHA}_{2} \mathrm{DS}_{2}$-VASc score. In the four pivotal studies 
comparing NOACs and warfarin in patients with nonvalvular AF, NOACs are at least as effective as warfarin to reduce stroke and at the same time are much safer alternatives in terms of life-threatening bleeding complications. Nonetheless, direct extrapolation of these results to other subgroups of AF patients may not be appropriate, given the different mechanisms of thrombus formation in different diseases. For instance, in the RE-ALIGN trial randomising 252 patients with recent mechanical valvular replacement in a 2:1 ratio to receive either dabigatran or warfarin, there was an excess of thromboembolic as well as bleeding events among patients randomised to dabigatran, rendering the study prematurely terminated. ${ }^{32}$

On the other hand, international guidelines do not recommend NOACs for patients with $\mathrm{AF}$ and moderate or severe MS due to the lack of reliable data from clinical trials. Nonetheless, it remains undetermined whether NOACs can be used as an alternative to warfarin for patients with $\mathrm{AF}$ and moderate or severe MS due to the lack of clinical trial. The current study has several important implications, particularly in Asian countries. First, while MS is now a rare condition in developed countries, it remains relatively prevalent in many Asian countries. In addition, the risk of stroke among patients with $\mathrm{AF}$ and MS is only second to those with mechanical valvular replacement ranging from $4 \%$ to $17 \%$. Second, previous epidemiological studies ${ }^{12-14} 213334$ and subanalyses of the pivotal NOAC trials ${ }^{15-17}$ have consistently reported a much higher nominal risk of ICH among Asians than non-Asians, favouring NOACs over warfarin therapy. More importantly, the notoriously poor TTR for warfarin in Asian populations observed in real-world data ${ }^{18}$ 34-38 and pivotal NOAC trials ${ }^{15-17}$ substantially undermines the overall clinical benefits of warfarin therapy. In fact, the annual incidence of ICH among patients with $\mathrm{AF}$ and MS treated with warfarin has been reported to be as high as $0.93 \%$ per year, ${ }^{10}$ urging a much safer alternative.

In the present study, the NOAC of choice is dabigatran, the first NOAC with an approved indication for stroke prevention in patients with non-valvular AF from the United States Food and Drug Administration in 2009. In the pivotal study, the RE-LY Study, ${ }^{39}$ patients with nonvalvular $\mathrm{AF}$ with $\mathrm{CHADS}_{2}>1$ were randomly assigned to two doses of dabigatran: $110 \mathrm{mg}$ or $150 \mathrm{mg}$ two times per day; or adjusted-dose warfarin. After a median follow-up of 2.0 years, the low-dose regime was found to be as effective as warfarin in preventing the primary endpoint (a composite of stroke and systemic embolism) (1.52\%/year vs $1.69 \%$ / year), but with a substantially lower risk of major bleeding and ICH. ${ }^{39}$ On the other hand, the standard dose dabigatran (150 mg two times per day) is superior to warfarin in reducing the primary composite endpoint and ICH, with a comparable risk of major bleeding. ${ }^{39}$ In an analysis comparing the effectiveness and safety of dabigatran according to the ethnicity of study participants, dabigatran appears to be more effective in stroke prevention as well as safer in terms of ICH compared with warfarin. This is in concordance to subsequent real-world cohorts of patients with AF from territory-wide registries from Asia Pacific region. Plausible explanations include suboptimal quality of warfarin therapy with low TTR and a higher risk of ICH in Asian populations. ${ }^{18} 23354041$ An additional reason for the choice of dabigatran in the present study is the wide availability of its antidote, idarucizumab in Asian countries, which provides extra-protection of patients in the clinical trial.

The study is designed to provide clinicians with robust, much-needed information regarding stroke prevention strategy for patients with $\mathrm{AF}$ and moderate or severe MS. The results will have immediate and long-term impacts on the management of these very high-risk patients with AF.

\section{Author affiliations}

${ }^{1}$ Cardiology Division, Department of Medicine, University of Hong Kong, Hong Kong, Hong Kong SAR, China

${ }^{2}$ Department of Pharmacology and Pharmacy, University of Hong Kong, Hong Kong, Hong Kong SAR, China

${ }^{3}$ Cardiology Division, Guangdong Cardiovascular Institute, Guangzhou, Guangdong, China

${ }^{4}$ Cardiology Division, South China University of Technology, Guangzhou, Guangdong, China

${ }^{5}$ Cardiology Division, Department of Medicine and Geriatrics, Ruttonjee and Tang Siu Kin Hospital, Hong Kong, Hong Kong SAR, China

${ }^{6}$ Cardiology Division, Department of Medicine and Geriatrics, Tuen Mun Hospital, Hong Kong, Hong Kong SAR, China

${ }^{7}$ Cardiac Medical Unit, Grantham Hospital, Hong Kong, Hong Kong SAR, China

${ }^{8}$ Cardiology Division, Department of Medicine \& Therapeutics, Chinese University of Hong Kong, Hong Kong, Hong Kong SAR, China

Contributors MZ, EWC, JJH, CKW, BPY and C-WS contributed to the conception and design of the study. MZ, EWC, JJH, CKW, Y-ML, DH, C-CL, C-CT, AYTW, ASYY, KKWC, YF, NT, JC, C-YY, K-LL, C-WC, HL, ANG, KF, M-HJ, YKH, BPY and C-WS contributed to the acquisition of data. Data analysis and interpretation will be conducted by MZ, EWC, JJH, CKW, BPY and C-WS. MZ, EWC, JJH, CKW, BPY and C-WS wrote the first draft of the protocol and revised the protocol critically for important intellectual content. All authors have read and approved the final version of the manuscript to be published.

Funding The authors have not declared a specific grant for this research from any funding agency in the public, commercial or not-for-profit sectors.

Competing interests None declared.

Patient consent for publication Not required.

Provenance and peer review Not commissioned; externally peer reviewed.

Open access This is an open access article distributed in accordance with the Creative Commons Attribution Non Commercial (CC BY-NC 4.0) license, which permits others to distribute, remix, adapt, build upon this work non-commercially, and license their derivative works on different terms, provided the original work is properly cited, appropriate credit is given, any changes made indicated, and the use is non-commercial. See: http://creativecommons.org/licenses/by-nc/4.0/.

\section{ORCID iDs}

Mi Zhou http://orcid.org/0000-0002-0864-9512

Chun Ka Wong http://orcid.org/0000-0001-5205-9440

\section{REFERENCES}

1 Lip GYH, Tse HF, Lane DA. Atrial fibrillation. Lancet 2012;379:648-61.

2 January CT, Wann LS, Calkins H, et al. 2019 AHA/ACC/HRS focused update of the 2014 AHA/ACC/HRS guideline for the management of patients with atrial fibrillation: a report of the American College of Cardiology/American heart association Task force on clinical practice guidelines and the heart rhythm society in collaboration with the Society of thoracic surgeons. Circulation 2019;140:e125-51. 
3 Kirchhof P, Benussi S, Kotecha D, et al. 2016 ESC guidelines for the management of atrial fibrillation developed in collaboration with EACTS. Europace 2016;18:1609-78.

4 Hart RG, Pearce LA, Aguilar MI. Meta-Analysis: antithrombotic therapy to prevent stroke in patients who have nonvalvular atrial fibrillation. Ann Intern Med 2007;146:857-67.

5 Siu C-W, Pong V, Zhang X, et al. Risk of ischemic stroke after newonset atrial fibrillation in patients with hyperthyroidism. Heart Rhythm 2009;6:169-73.

6 Guo Y, Wang H, Tian Y, et al. Multiple risk factors and ischaemic stroke in the elderly Asian population with and without atrial fibrillation. An analysis of 425,600 Chinese individuals without prior stroke. Thromb Haemost 2016;115:184-92.

7 Chandrashekhar Y, Westaby S, Narula J. Mitral stenosis. Lancet 2009;374:1271-83.

8 De Caterina R, John Camm A. Non-Vitamin K antagonist oral anticoagulants in atrial fibrillation accompanying mitral stenosis: the concept for a trial. Europace 2016;18:6-11.

9 Breithardt G, Baumgartner H, Berkowitz SD, et al. Clinical characteristics and outcomes with rivaroxaban vs. warfarin in patients with non-valvular atrial fibrillation but underlying native mitral and aortic valve disease participating in the rocket $\mathrm{AF}$ trial. Eur Heart J 2014;35:3377-85.

$10 \mathrm{Kim}$ JY, Kim S-H, Myong J-P, et al. Outcomes of direct oral anticoagulants in patients with mitral stenosis. J Am Coll Cardiol 2019;73:1123-31.

11 Giugliano RP, O'Gara PT. DOACs in patients with mitral stenosis and atrial fibrillation: time for a randomized clinical trial. J Am Coll Cardiol 2019;73:1132-4.

12 Tse H-F, Wang Y-J, Ahmed Ai-Abdullah M, et al. Stroke prevention in atrial fibrillation--an Asian stroke perspective. Heart Rhythm 2013;10:1082-8.

13 Chong B-H, Chan K-H, Pong V, et al. Use of aspirin in Chinese after recovery from primary intracranial haemorrhage. Thromb Haemost 2012;107:241-7.

14 Friberg L, Rosenqvist M, Lip GYH. Evaluation of risk stratification schemes for ischaemic stroke and bleeding in 182678 patients with atrial fibrillation: the Swedish atrial fibrillation cohort study. Eur Heart J 2012;33:ehr488:1500-10.

15 Hori M, Connolly SJ, Zhu J, et al. Efficacy and safety of dabigatran versus warfarin in patients with atrial fibrillation: analysis in Asian population in RE-LY trial. Cerebrovascular Disease 2012;34.

16 Hankey GJ, Stevens S, Piccini JP, et al. Predictors of intracranial hemorrhage among anticoagulated patients with atrial fibrillation: insights from the rivaroxaban once daily oral direct factor $\mathrm{Xa}$ inhibition compared with vitamin $\mathrm{K}$ antagonism for prevention of stroke and embolism trial in atrial fibrillation (rocket AF). Stroke 2012;43:A152

17 Tanahashi N, Hori M, Matsumoto M, et al. Rivaroxaban versus warfarin in Japanese patients with nonvalvular atrial fibrillation for the secondary prevention of stroke: a subgroup analysis of J-ROCKET AF. J Stroke Cerebrovasc Dis 2013;22:S1052-3057(12)004375:1317-25.

18 Chan P-H, Huang D, Lau C-P, et al. Net Clinical Benefit of Dabigatran Over Warfarin in Patients With Atrial Fibrillation Stratified by ${ }_{\text {CHAZDS2 }}$-VASc and Time in Therapeutic Range. Can J Cardiol 2016;32:1247:1247.e15-1247.e21.

19 Chan P-H, Li W-H, Hai J-J, et al. Gastrointestinal haemorrhage in atrial fibrillation patients: impact of quality of anticoagulation control. Eur Heart J Cardiovasc Pharmacother 2015;1:265-72.

20 Lee Y-K, Lau Y-M, Cai Z-J, et al. Modeling treatment response for lamin A/C related dilated cardiomyopathy in human induced pluripotent stem cells. J Am Heart Assoc 2017;6:005677.

21 Lau WCY, Li X, Wong ICK, et al. Bleeding-related hospital admissions and 30-day readmissions in patients with non-valvular atrial fibrillation treated with dabigatran versus warfarin. $J$ Thromb Haemost 2017:15:1923-33.

22 Huang D, Cheng Y-Y, Chan P-H, et al. Rationale and design of the screening of pulmonary hypertension in systemic lupus erythematosus (SOPHIE) study. ERJ Open Res 2018;4:00135-2017.

23 Qi X, Wong BL, Lau SH, et al. A hemoglobin-based oxygen carrier sensitized cisplatin based chemotherapy in hepatocellular carcinoma. Oncotarget 2017;8:85311-25.

24 Chan A-W, Tetzlaff JM, Altman DG, et al. Spirit 2013 statement: defining standard protocol items for clinical trials. Ann Intern Med 2013;158:200-7.

25 Chan A-W, Tetzlaff JM, Gøtzsche PC, et al. Spirit 2013 explanation and elaboration: guidance for protocols of clinical trials. $B M J$ 2013;346:e7586.

26 Altman DG, Schulz KF, Moher D, et al. The revised consort statement for reporting randomized trials: explanation and elaboration. Ann Intern Med 2001;134:663-94.

27 Moher D, Schulz KF, Altman D, et al. The CONSORT statement: revised recommendations for improving the quality of reports of parallel-group randomized trials 2001. Explore 2005;1:40-5.

28 Cockcroft DW, Gault MH. Prediction of creatinine clearance from serum creatinine. Nephron 1976;16:31-41.

29 Pugh RN, Murray-Lyon IM, Dawson JL, et al. Transection of the oesophagus for bleeding oesophageal varices. $\mathrm{Br} J$ Surg 1973;60:646-9.

30 Rosendaal FR, Cannegieter SC, van der Meer FJ, et al. A method to determine the optimal intensity of oral anticoagulant therapy. Thromb Haemost 1993;69:236-9.

31 Camm AJ, Kirchhof P, Lip GYH, et al. Guidelines for the management of atrial fibrillation: the task force for the management of atrial fibrillation of the European Society of cardiology (ESC). Europace 2010;12:euq350:1360-420.

32 Eikelboom JW, Connolly SJ, Brueckmann M, et al. Dabigatran versus warfarin in patients with mechanical heart valves. $N$ Engl $J$ Med 2013;369:1206-14

33 Chan EW, Lau WCY, Siu CW, et al. Effect of suboptimal anticoagulation treatment with antiplatelet therapy and warfarin on clinical outcomes in patients with nonvalvular atrial fibrillation: a population-wide cohort study. Heart Rhythm 2016;13:1581-8.

34 Teo KC, Mahboobani NR, Lee R, et al. Warfarin associated intracerebral hemorrhage in Hong Kong Chinese. Neurol Res 2014;36:143-9.

35 Huang D, Wong C-L, Cheng K-W, et al. Impact of provision of time in therapeutic range value on anticoagulation management in atrial fibrillation patients on warfarin. Postgrad Med J 2018;94:207-11.

36 Chan PH, Hai JJ, Chan EW, et al. Use of the SAMe-TT2R2 score to predict good anticoagulation control with warfarin in Chinese patients with atrial fibrillation: relationship to ischemic stroke incidence. PLoS One 2016;11:e0150674.

37 Ho C-W, Ho M-H, Chan P-H, et al. Ischemic stroke and intracranial hemorrhage with aspirin, dabigatran, and warfarin: impact of quality of anticoagulation control. Stroke 2015;46:23-30.

38 Siu C-W, Tse H-F. Net clinical benefit of warfarin therapy in elderly Chinese patients with atrial fibrillation. Circ Arrhythm Electrophysiol 2014:7:300-6.

39 Connolly SJ, Ezekowitz MD, Yusuf S, et al. Dabigatran versus warfarin in patients with atrial fibrillation. $N$ Engl J Med 2009;361:1139-51.

40 Hai J-J, Chan P-H, Chan Y-H, et al. Prediction of thromboembolic events in heart failure patients in sinus rhythm: the Hong Kong heart failure registry. PLoS One 2016;11:e0169095.

41 Chan P-H, Li W-H, Hai J-J, et al. Time in therapeutic range and percentage of international normalized ratio in the therapeutic range as a measure of quality of anticoagulation control in patients with atrial fibrillation. Can J Cardiol 2016;32:1247.e23-1247.e28. 